\title{
Factores de riesgo asociados a la seroprevalencia de cisticercosis en el Municipio de Mitú, Colombia
}

\author{
Carmen Elena Rincón Valenzuela1', Astrid Carolina Flórez Sánchez \\ Instituto Nacional de Salud, Bogotá D.C., Colombia \\ Correspondencia: crincon@ins.gov.co
}

Recibido: 11-08-09 / Aceptado: 23-10-09

\section{Resumen}

La cisticercosis es una enfermedad de salud pública que afecta a todas las personas sin discriminación de género ni edad, por eso es necesario considerar estrategias que permitan su erradicación. Una forma de lograrlo es la creación de campañas nacionales, que permitan mejoras en los servicios sanitarios para interrumpir el ciclo de vida de la Taenia solium. También es fundamental incluir aspectos educacionales donde se considere información médica y veterinaria. El objetivo de este estudio fue conocer los posibles factores de riesgo relacionados con la presencia de cisticercosis en la población del municipio de Mitú, Vaupés, Colombia, que ayuden a identificar oportunamente la cadena de transmisión de esta enfermedad.

Se encuestaron 1.141 personas de las cuales 44 I resultaron positivas para lgG cisticerco mediante la técnica de Elisa. El 56,0\% fueron mujeres y principalmente el grupo de 19 a 40 años de edad fue el de mayor porcentaje de seroprevalencia. La falta de hábitos higiénicos, como el lavado de manos después de salir del baño, presentó un mayor riesgo de infección por cisticercosis con respecto aquellos que sí lo realizaban (OR=I, I 9 IC 95\%, I,03 - I,38). También se halló un alto porcentaje de falta de hábitos de higiene entre las personas seropositivas con respecto al consumo de carne de cerdo.

Palabras clave: cisticercosis, epidemiología, inmunoglobulina G, neurocisticercosis, Taenia solium.

$$
\begin{aligned}
& \text { Abstract } \\
& \text { Risk factors associated with seroprevalence of cysticercosis in the municipality of } \\
& \text { Mitú, Colombia } \\
& \text { Cysticercosis is a public health disease that affects all people without discrimination of gender or age, } \\
& \text { so it is necessary to consider strategies for its eradication. One way is to create national campaigns that } \\
& \text { enable improvements in health services to interrupt the life cycle of Taenia solium. It is also essential to } \\
& \text { include educational aspects where medical and veterinary information is considered. The aim of this } \\
& \text { study was to identify possible risk factors related to the presence of cysticercosis in the population of the } \\
& \text { town of Mitú, Vaupes, Colombia, to help appropriately identify the chain of transmission of this disease. } \\
& \text { Out of I, I I I surveyed people, } 44 \text { I were positive for cysticercus IgG by ELISA. 56.0\% were women } \\
& \text { and especially the group of I } 9 \text {-40 years old had the highest percentage of positive results. The lack } \\
& \text { of hygienic habits, like washing hands after leaving the bathroom, had a higher risk of infection with }
\end{aligned}
$$


cysticercosis with respect to those who did washed $(\mathrm{OR}=1.1995 \% \mathrm{Cl}, 1.03$ to I.38). Also, a high percentage of lack of hygiene habits was found among people with HIV in relation to pork consumption.

Keywords: cysticercosis, epidemiology, immunoglobulin G, neurocysticercosis, Taenia solium.

\section{Introducción}

La cisticercosis porcina constituye un problema de salud pública común en países en vías de desarrollo (1). Las condiciones socioeconómicas, de salubridad y de educación sanitaria existentes en estos países favorecen la permanencia y transmisión de la Taenia solium (2). La crianza de porcinos es una actividad de importancia económica para los pobladores andinos, que además de brindar alimento, puede desarrollarse de manera extensiva o al pastoreo con baja inversión (3). Sin embargo, la infección de los cerdos trae consigo importantes pérdidas para la precaria economía del campesino debido a la merma en precio de la carne infectada o al decomiso total.

La infección de neurocisticercosis ocurre cuando los huevos de $T$. solium son ingeridos, estos son incubados en el duodeno, los embriones invaden la mucosa intestinal y causan lesiones en el aparato gastrointestinal, diseminándose por el torrente sanguíneo. Las localizaciones más comunes del cisticerco en su hospedero humano son: el esqueleto, los músculos y el cerebro, este puede sobrevivir por un lapso de hasta 10 años en los tejidos sin causar sintomatología aparente (4).

En ciertas regiones de Colombia, la neurocisticercosis es endémica, especialmente donde la disposición de excretas humanas es inadecuada, no existe todavía un programa de notificación que revele el panorama de esta enfermedad y sus posibles factores de riesgo, tan solo se han realizado algunos estudios aislados en diferentes partes y poblaciones del país, demostrándose serologías positivas para cisticercosis en el 25\% de pacientes con convulsiones (Nariño y Antioquia), en el $13 \%$ de los pacientes admitidos a un servicio clínico de neurología (Cali, Valle del Cauca), y en un $10 \%$ de una población endémica para esta infección (El Hato, Santander) (5-7).
Es importante contribuir al conocimiento de los posibles factores de riesgo relacionados con la presencia de cisticercosis presente en la población del municipio de Mitú-Vaupés, que permita identificar a tiempo la cadena de transmisión de esta enfermedad, catalogada actualmente como un problema de salud pública.

\section{Materiales y métodos}

Se llevó a cabo un estudio descriptivo de corte trasversal durante el mes de mayo del 2009. Se encuestaron 1140 personas localizadas en la zona urbana, suburbana y rural del municipio de Mitú, Vaupés, Colombia, con el fin de obtener datos generales relacionados con el estado civil, escolaridad, afiliación al sistema general de seguridad social, ocupación, consumo de carne de cerdo, hábitos higiénicos, estado de salud, sintomatología compatible con la enfermedad, servicios sanitarios y condiciones de la vivienda.

Adicionalmente, a las personas encuestadas, además de su participación voluntaria previa firma del consentimiento informado, se les tomó una muestra de sangre en tubo seco, las cuales fueron enviadas al Laboratorio de Parasitología del Instituto Nacional de Salud para la determinación de anticuerpos IgG anti-cisticerco, mediante la técnica de Elisa. La información recolectada fue consignada en una base de datos creada en Access 2007 y analizada en EpiInfo versión 3.5.1.

\section{Resultados}

De los 1140 pacientes, 441 (38,7\%) fueron seropositivos para cisticercosis, $247(56,0 \%)$ mujeres y $194(44,0 \%)$ hombres, de estos $280(63,4 \%)$ habitaban en zona urbana, $128(29,0 \%)$ en zona 
suburbana y $33(7,4 \%)$ en zona rural. En la Tabla 1 se relaciona la distribución de la población seropositiva por municipios y veredas, y en la Tabla 2 por grupos de edades.

Tabla 1. Distribución de las muestras seropositivas por centros poblados y veredas del municipio de Mitú, Vaupés.

\begin{tabular}{|c|c|}
\hline MUNICIPIOS Y VEREDAS & $(\mathbf{n}) \%$ \\
\hline Mitú & 29466,6 \\
\hline Acaricuara & 337,48 \\
\hline Bocas del Yi & 81,81 \\
\hline Camutí & 112,49 \\
\hline Macaquiño & 132,94 \\
\hline Mirití & 92,04 \\
\hline Mituseño & 102,26 \\
\hline Pirasemo & 132,94 \\
\hline San Juan de Cucurá & 30,68 \\
\hline Ceima Cachivera & 102,26 \\
\hline Tapurucuara & 163,62 \\
\hline Tucunaré & 92,04 \\
\hline Santa Helena & 122,72 \\
\hline TOTAL & 441100 \\
\hline
\end{tabular}

Tabla 2. Distribución de casos positivos por grupos de edades.

\begin{tabular}{|c|c|c|}
\hline $\begin{array}{c}\text { GRUPOS DE } \\
\text { EDAD }\end{array}$ & $(\mathbf{n})$ & $\%$ \\
\hline 2 a 10 años & 17 & 3,9 \\
\hline II a 18 años & 43 & 9,8 \\
\hline I9 a 40 años & 221 & 50 \\
\hline 4I a 64 años & 160 & 36,3 \\
\hline TOTAL & 441 & 100 \\
\hline
\end{tabular}

Con respecto a la escolaridad, se observó que el mayor porcentaje de personas seropositivas tenían secundaria incompleta $130(29,4 \%)$, seguido de primaria completa $87(19,7 \%)$, de primaria incompleta $78(17,6 \%)$, universidad completa 13 $(2,9 \%)$ y con estudios de posgrado $2(0,5 \%)$. En cuanto al estrato socioeconómico, 375 (85,0\%) de los seropositivos pertenecían al estrato uno, 64 (14,5\%) al estrato dos y $2(0,4 \%)$ al estrato tres.
$\mathrm{Al}$ realizar la asociación entre el consumo de carne de cerdo con los seropositivos se encontró que 317 $(71,9 \%)$ la incluyen dentro de su dieta, mientras que $124(28,1 \%)$ no la consumen, sin embargo no se demostró riesgo estadísticamente significativo $(\mathrm{OR}=0.65 \mathrm{IC}$ 95\%, 0.49-0.86). El lavado de manos después de salir del baño fue otras de las variables incluida, encontrándose que $205(46,5 \%)$ de las personas positivas para cisticercosis se lavaban siempre las manos, pero $236(53,5 \%)$ ocasionalmente o nunca lo realizaba, identificándose un mayor riesgo a la infección por cisticercosis en este último grupo (OR=1,19 IC 95\%, 1,03-1,38).

En los seropositivos existe un mayor riesgo al dolor de cabeza como un síntoma asociado a la cisticercosis $(\mathrm{OR}=1,41 \mathrm{IC} 95 \%, 1,10-1,80)$. En la Tabla 3 se relacionan las sintomatologías reportadas por las personas que participaron en este estudio.

Tabla 3. Sintomatología presente en los pacientes seropositivos.

\begin{tabular}{|l|l|l|}
\hline SINTOMATOLOGIA & $(\mathbf{n})$ & $\%$ \\
\hline Convulsiones & 10 & 2,26 \\
\hline Dolor de cabeza & 239 & $54, \mathrm{I}$ \\
\hline Desmayos & 19 & 4,30 \\
\hline Mareos & 76 & 17,2 \\
\hline Trastornos visuales & 39 & 8,84 \\
\hline Diarrea & 58 & $13, \mathrm{I}$ \\
\hline TOTAL & $44 I$ & 100 \\
\hline
\end{tabular}

La obtención de agua que surte la vivienda permitió demostrar que no existe suministro de agua potable y que el 59,2\% la obtienen únicamente de aguas lluvias, el 21,3\% del rio y de aguas lluvias y el 16,3\% únicamente del rio. La expulsión de fragmentos de parásitos por las personas encuestadas fue otro hallazgo, donde el 56,0\% (247) respondió que había expulsado parásitos por materia fecal y 43,9\% (194) no había expulsado ó manifestaron no saber o no darse cuenta, identificándose un mayor riesgo a la infección por cisticercosis en aquellos que expulsaban parásitos $(\mathrm{OR}=1,15$ IC 95\%, 0,89-1,47). 


\section{Discusión}

La prevalencia del $38.7 \%$ encontrada en la población del municipio de Mitú, Vaupés, Colombia, para cisticercosis fue alta, comparada con un estudio realizado en Morelos, México, que fue del 10.8\% (9). Los seropositivos presentaron dolor de cabeza como síntoma asociado a la neurocisticercosis, hallazgo también encontrado en el estudio realizado a comerciantes de alimentos en mercados en la ciudad de México, donde refirieron presentar no sólo dolor de cabeza sino también desmayos y ataques epilépticos (10).

De acuerdo a diversos estudios, se ha establecido que el bajo nivel educativo también se encuentra fuertemente asociado no solamente a la presencia de teniosis/cisticercosis sino a muchas otras parasitosis. En cuanto a los factores socioeconómicos, la seroprevalencia de la cisticercosis claramente identifica como potencialmente riesgosos a todos aquéllos relacionados con el subdesarrollo social, deficiente escolaridad, vivienda en malas condiciones, ruralidad, hacinamiento, pero también señala enfáticamente que en México el riesgo de contacto con T. solium no es despreciable ni para los estratos sociales más privilegiados $(11,12)$.

En las personas seropositivas tanto el género y la edad fueron similares con los resultados encontrados en el estudio de seroepidemiología de la cisticercosis en México, donde el mayor porcentaje de prevalencia de esta enfermedad fue en las mujeres y en las edades tempranas y tardías. Esto se puede atribuir a que los nińos tienen mayor contacto con el suelo contaminado, junto con las personas de mayor edad, quienes permanecen mayor tiempo y de forma más continua en la vivienda, exponiéndose a condiciones que propician el contacto (12).

El consumo de carne de cerdo por las personas seropositivas fue similar a los resultados hallados por los investigadores de La Merced, los cuales encontraron que el $65,5 \%$ de los encuestados aceptaban haber comido carne de cerdo, por su mejor sabor (13), sin embargo en este estudio no se evidenció riesgo en los seropositivos relacionado con el consumo de carne de cerdo. Es importante tener en cuenta que se presentó mayor riesgo entre las personas que habían manifestado expulsar parásitos y la presencia de anticuerpos anti-cisticerco, siendo la presencia de teniósicos o portadores de Taenia un factor de riesgo para la transmisión de la enfermedad. Este hallazgo fue más relevante comparado con el estudio de prevalencia en el departamento de Caldas, en el cual se halló un porcentaje bajo del 2,3\% con respecto a la expulsión de proglótides (14).

En cuanto a hábitos higiénicos en los seropositivos, definitivamente en este estudio el lavado de manos después de la evacuación de heces fecales en letrinas, sanitarios e inclusive al aire libre, constituye un factor de riesgo desencadenante de la transmisión de la cisticercosis, ya que son los huevos del parásito los que pueden contaminar alimentos y bebidas.

La cisticercosis actualmente es una enfermedad de interés en salud pública que afecta a todas las personas sin discriminar género ni edad, por eso se debe considerar estrategias que permitan la prevención de esta enfermedad, como la creación de campañas nacionales, que incluyan aspectos educacionales, médicos, veterinarios y mejoras en los servicios sanitarios que estén diseñados para interrumpir el ciclo de vida de la T. solium.

\section{Referencias}

1. Acha P, Szyfres B. Zoonosis y enfermedades transmisibles comunes al hombre y a los animales. 2a ed. Publicación Científica $N^{\circ} 503$ OPS. 1992. p. 763- 773.

2. Evans C, García H, Gilman R. Cysticercosis. En: Hunter's tropical medicine and emerging infectious diseases. 8th ed. W.B. Sander Co. Phyladelphia. 2000.

3. García H, Martínez M, Gilman R, Herrera G, Tsang V, Pilcher J. et al. Cysticercosis as a major cause of epilepsy in Peru. The Cysticercosis Working Group in Perú (CWG). Lancet. 1991;338:549-351.

4. Imirizaldu L, Miranda L, García-Gurtubay I, Gastón I, Urriza J, Quesada P. Neurocisticercosis. Una enfermedad emergente. An Sist Sanit Navar. 2004;27: 201-209.

5. Arango J, Roldan E, Vargas H, Vásquez F, Vélez G, Villegas A, Ocampo N, Botero D, Estrada J. Condiciones higiénicas, anticuerpos humanos y porcinos contra cysticercus cellulosae en 40 familias del municipio de San Vicente (Antioquia). Rev Med UPB.1998.

6. Franco S, Hincapié M, Mejía O, Botero D. Estudio epidemiológico de epilepsia y neurocisticercosis. Rev Med UIS. 1986;14:143-174.

7. Palacio G, Tobón M, Mora O, Sánchez J, Jiménez M. Prevalencia de Neurocisticercosis en individuos afectados de epilepsia. Revista Neurol. 1997; 25:1406-1410.

8. Ayvar V, González A, Falcón N, Bernal T, Mena C. Seroprevalencia de la cisticercosis porcina en tres Caseríos de la provincia de Andahuaylas, Apurímac. Rev Inv Vet Perú 2004;15:56-62.

9. Rosas G, Fragoso G. Factores de Riesgo y Prevalencia de la Cisticercosis en Morelos. Narraciones de la Ciencia. 2009;63-69. 
10. Cruz V, Plancarte A, Moran I, Valencia S, Rodríguez G, Vega L. Teniosis y cisticercosis en comerciantes de alimentos en mercados de un área de la ciudad de México. Parasitol Latinoam. 2003;58:41-48.

11. Moran I, Cruz V. Teniosis-cisticercosis. Epidemiologia y factores de riesgo. Rev Fac Med UNAM 2000;43:32-33.

12. Larralde C, Padilla A, Hernández M, Govezensky T, Sciutto E, Gutiérrez G. et al. Seroepidemiología de la Cisticercosis en México. Salud Pública México. 1992;34:197-210.
13. Cock B, Hdo O. Comportamiento de los factores de riesgo asociados a Cisticercosis Humana en «La Merced»: Tesis de grado, Facultad de Veterinaria, Universidad de Caldas, 1.990.

14. Muñoz A. Neurocisticercosis. Aspectos Clínicos Prevalencia en el Departamento de Caldas. Archivos de Medicina. Universidad de Caldas. 2005; 14-27. 